\title{
Genomic mid-range inhomogeneity correlates with an abundance of RNA secondary structures
}

\author{
Jason M Bechtel ${ }^{\dagger 1}$, Thomas Wittenschlaeger ${ }^{\dagger 1,6}$, Trisha Dwyer ${ }^{1,2}$, \\ Jun Song1,2,7, Sasi Arunachalam1,3, Sadeesh K Ramakrishnan 1,4, \\ Samuel Shepard 1,5 and Alexei Fedorov*1,5
}

\begin{abstract}
Address: ${ }^{1}$ Program in Bioinformatics and Proteomics/Genomics, University of Toledo Health Science Campus, Toledo, OH 43614, USA, ${ }^{2}$ Dept. of Physiology and Pharmacology, University of Toledo Health Science Campus, Toledo, OH 43614, USA, ${ }^{3}$ Dept. of Neuroscience, University of Toledo Health Science Campus, Toledo, OH 43614, USA, ${ }^{4}$ Department of Cardiovascular and Metabolic Diseases, University of Toledo Health Science Campus, Toledo, OH 43614, USA, 5 Dept. of Medicine, University of Toledo Health Science Campus, Toledo, OH 43614, USA, ${ }^{6}$ Department of Biological Sciences, Bowling Green State University, Bowling Green, OH 43403, USA and ${ }^{7}$ Luye Pharmaceutical LTD, Rm1107, Zhubang 2000 Business Center, Chaoyang District, Beijing 100025, PR China
\end{abstract}

Email: Jason M Bechtel - jason.bechtel@utoledo.edu; Thomas Wittenschlaeger - tmwitten@gmail.com;

Trisha Dwyer - Trisha.Dwyer@utoledo.edu; Jun Song - songjun@luye-pharm.com; Sasi Arunachalam - sasi.arunachalam@utoledo.edu; Sadeesh K Ramakrishnan - sadeesh.ramakrishnan@utoledo.edu; Samuel Shepard - Samuel.Shepard@utoledo.edu;

Alexei Fedorov* - alexei.fedorov@utoledo.edu

* Corresponding author †Equal contributors

Published: 12 June 2008

BMC Genomics 2008, 9:284 doi:|0.| | 86/|47|-2|64-9-284
Received: 2I February 2008

Accepted: 12 June 2008

This article is available from: http://www.biomedcentral.com/I47I-2/64/9/284

(C) 2008 Bechtel et al; licensee BioMed Central Ltd.

This is an Open Access article distributed under the terms of the Creative Commons Attribution License (http://creativecommons.org/licenses/by/2.0), which permits unrestricted use, distribution, and reproduction in any medium, provided the original work is properly cited.

\begin{abstract}
Background: Genomes possess different levels of non-randomness, in particular, an inhomogeneity in their nucleotide composition. Inhomogeneity is manifest from the short-range where neighboring nucleotides influence the choice of base at a site, to the long-range, commonly known as isochores, where a particular base composition can span millions of nucleotides. A separate genomic issue that has yet to be thoroughly elucidated is the role that RNA secondary structure (SS) plays in gene expression.

Results: We present novel data and approaches that show that a mid-range inhomogeneity $(\sim 30$ to 1000 nt) not only exists in mammalian genomes but is also significantly associated with strong RNA SS. A wholegenome bioinformatics investigation of local SS in a set of 11,315 non-redundant human pre-mRNA sequences has been carried out. Four distinct components of these molecules (5'-UTRs, exons, introns and $3^{\prime}-$ UTRs) were considered separately, since they differ in overall nucleotide composition, sequence motifs and periodicities. For each pre-mRNA component, the abundance of strong local SS $(<-25 \mathrm{kcal} /$ $\mathrm{mol}$ ) was a factor of two to ten greater than a random expectation model. The randomization process preserves the short-range inhomogeneity of the corresponding natural sequences, thus, eliminating shortrange signals as possible contributors to any observed phenomena.

Conclusion: We demonstrate that the excess of strong local SS in pre-mRNAs is linked to the little explored phenomenon of genomic mid-range inhomogeneity (MRI). MRI is an interdependence between nucleotide choice and base composition over a distance of 20-1000 nt. Additionally, we have created a public computational resource to support further study of genomic MRI.
\end{abstract}




\section{Background \\ RNA secondary structures}

Secondary structures (SS) are crucial elements for the biosynthesis and/or correct action of non-coding RNAs in mammals and other eukaryotes. Moreover, they are key regulators in the function and turnover of mRNA molecules. SS in pre-mRNAs regulate the splicing process [1-3]. In mature mRNAs, SS located in 5'-untranslated regions (5'-UTRs) signal for translational control [4,5] and those located in 3'-untranslated regions (3'-UTRs) regulate subcellular localization and stability [6-8]. SS located inside protein-coding sequences could play a role in translational speed and stability $[9,10]$.

Prior studies of the strength of computer-predicted SS in mRNA have had conflicting conclusions [11,12]. More importantly, these studies did not investigate the abundance of SS and considered only coding sequences. This spurred us to perform a bioinformatics investigation into the abundance of SS throughout mammalian genomes. Our results show that the existence of many energeticallystrong SS is associated with the phenomenon of global mid-range inhomogeneity (MRI), manifest as nucleotide compositional relationships at a scale of 20 to 1000 bases throughout the genome. MRI appears as a strong tendency for the clustering of particular bases (e.g. C and G nucleotides, or $\mathrm{G}$ and $\mathrm{A}$ nucleotides) inside short regions of genomic sequences. This paper provides new approaches and tools to gain insights into this form of genomic inhomogeneity.

\section{Short-range inhomogeneity}

It is well established that the particular base $(A, G, C$, or T) that appears in a given position of a genomic sequence significantly depends upon the nearest bases surrounding its position $[13,14]$. Consequently, the frequency $(F)$ of a dinucleotide $X Y$ is often not equal to the product of the individual frequencies of nucleotides $X$ and $Y\left(\mathrm{~F}_{X Y} \neq \mathrm{F}_{X}\right.$ ${ }^{*} \mathrm{~F}_{Y}$ ). The highest interdependence of base frequencies is always observed for adjacent nucleotides. The ratio ( $\mathrm{F}_{\mathrm{XY}} /$ $\left(\mathrm{F}_{\mathrm{X}}{ }^{*} \mathrm{~F}_{\mathrm{Y}}\right)$ ) for adjacent bases $X$ and $Y$ is known as a "genomic signature" [14]. Genomic signatures as low as 0.22 (for the CG dinucleotide in mouse) and as high as 1.75 (for the GC dinucleotide in Campylobacter jejuni) have been recorded [15]. The interdependence of base frequencies sharply drops with increasing distance. When the distance between nucleotides $X$ and $Y$ is more than six bases, their occurrence interdependency becomes negligible. Here, we refer to this type of interdependency between nucleotides separated from each other by a few positions as short-range inhomogeneity (SRI).

\section{Long-range inhomogeneity}

Also well recognized are long-range interdependencies in nucleotide frequencies on a scale of up to millions of bases, known as genomic isochores [16]. It has been shown that isochores can be generally categorized according to their level of $\mathrm{G}+\mathrm{C}$ content. Isochores defined by $\mathrm{G}+\mathrm{C}$ content correspond to many other genomic phenomena. GCrich isochores replicate later in S-phase, contain higher concentrations of genes, and have genes with shorter introns and untranslated regions. Moreover, GC-rich isochores tend to have an "open" chromatin structure and thus have higher rates of transcription [17]. Higher $\mathrm{G}+\mathrm{C}$ content isochores also experience higher recombination rates - perhaps lending support to the notion that higher recombination rates led to the creation of isochores through biased gene conversion [18]. While the evolution and maintenance of isochores is subject to debate, their presence is indeed evidence of existing interdependencies in nucleotide composition on the scale of tens of thousands to millions of nucleotides. We will refer to this form of non-randomness in genomic nucleotide composition as long-range inhomogeneity.

\section{Mid-range inhomogeneity}

The compositional non-randomness between the two extremes described above we call mid-range inhomogeneity or MRI. MRI has yet to be thoroughly investigated. The only well-known manifestation of mid-range inhomogeneity is CpG islands. Most attempts to define CpG islands set hard requirements for region size (at least 200 or 500 bases long), $\mathrm{G}+\mathrm{C}$ content (> $50 \%$ or $55 \%$ ), and $\mathrm{CpG}$ observed/expected ratio (>0.6 or 0.65 ) [[19,20], respectively]. CpG-islands are found near $60 \%$ of human genes, including all housekeeping genes and about half of the tissue-specific genes [21]. Here we demonstrate that MRI can be observed for regions from 30-1000 bp and is significant not only for $\mathrm{G}+\mathrm{C}$ content but for other nucleotide pairings $(A+G$ and $G+T)$ as well as for the individual bases.

\section{Results}

\section{Analysis of strong local SS within pre-mRNAs}

Distinct parts of mRNAs and introns have large variations in nucleotide composition (from 35\% to $60 \%$ of GC-content, see Table 1). Due to this difference the analyses of SS distribution were performed on four separate regions: GCrich 5'-UTR regions, GC-poor introns and 3'-UTR regions, and intermediate GC-content protein-coding regions of mRNAs. In addition, vertebrate and invertebrate species have considerable variations in their mRNA nucleotide composition. Within the mammalian class, however, the variation in GC-content is negligible (Table 1). For this reason we demonstrate only results for human sequences, although the observed trends are applicable to all mammals.

We begin by looking at SS created by the interactions of nucleotides less than 50 bases apart (local SS). Prediction 
Table I: Percentage of GC-composition in different regions of pre-mRNA for diverse animal species.

\begin{tabular}{lllll}
\hline SPECIES & \multicolumn{4}{c}{$\%$ GC-content } \\
\hline & 5'-UTRs & CDS & 3'-UTRs & Introns \\
\hline Human & $60 \%$ & $52 \%$ & $44 \%$ & $41 \%$ \\
Mouse & 59 & 52 & 44 & 43 \\
Cow & 60 & 54 & 44 & 43 \\
Chicken & 57 & 51 & 41 & 41 \\
Zebrafish & 45 & 50 & 37 & 35 \\
Drosophila & 45 & 54 & 36 & 40 \\
\hline
\end{tabular}

of local SS is more reliable than prediction of global RNA structures, which can span hundreds of nucleotides [22]. We examined these structures in 11,315 non-redundant human gene sequences (see Methods section) and calculated their strengths using the RNALfold program of the Vienna RNA package [23]. Figure 1 illustrates the distribution of local SS according to folding strength in distinct parts of mRNAs and introns. We concentrate our study on the stronger secondary structures. Such strong local SS could withstand competition with the many RNA-binding proteins that cover mRNAs and, thus, are more likely to persist in vivo. Indeed, hairpin structures with an mfe of $30 \mathrm{kcal} / \mathrm{mol}$ situated close to the mRNA cap significantly impede ribosome scanning, while hairpin structures with an mfe upwards of $-50 \mathrm{kcal} / \mathrm{mol}$ inhibit elongation [5]. Strong secondary structures are also required for the regulation of splicing. Kishore and Stamm showed that the 18 nt-long HBII-52 snoRNA antisense element interacts with a pre-mRNA segment with an $\mathrm{mfe}$ of $-27.6 \mathrm{kcal} / \mathrm{mol}$ and thereby determines the fate of alternative splicing in the serotonin receptor gene [24]. Finally, a majority of human miRNA genes from the microRNAdb [25] have strong interaction energies $(<-25 \mathrm{kcal} / \mathrm{mol})$ with dozens of tar- gets within mRNAs. Thus, "strong" local SS is defined as having a minimum free energy ( $\mathrm{mfe}$ ) value of less than or equal to $-25 \mathrm{kcal} / \mathrm{mol}$, so as to encompass all known functional local SS.

\section{Analysis of strong local SS in randomized sequences}

To evaluate the abundance of local SS, one must compare their prevalence in naturally occurring mRNAs with their levels in reference sequences having no selection for SS. In most research, reference sequences are randomly generated to have nucleotide compositions approximating those of the naturally occurring mRNAs. In order to properly compare local SS in natural and randomized sequences one needs to preserve short-range inhomogeneity (SRI), as discussed previously by Workman and Krogh [12].

Thus, to most accurately preserve SRI in randomized sequences we created a public resource for generating randomized sequences while taking into account the SRI of a given set of natural sequences. Our algorithm can take into account not only relative dinucleotide frequencies, but also frequencies of longer oligonucleotides (up to 9mers). We first applied our SRI-analyzer program (see Methods section) [26] to a set of natural mRNA sequences to obtain their oligonucleotide composition, shown in Table 2. Our second program, SRI-generator [26], then uses these oligonucleotide frequency tables to generate random sequences with approximately the same oligonucleotide distribution as the natural sequences but without any similarity in their sequence alignments. Table $2 \mathrm{dem}-$ onstrates the oligonucleotide frequencies for human 5'UTRs and two independent SRI-generated sets of sequences. Notably, the oligonucleotide compositions of the SRI-generated sequences are very close to those of the natural sequences, with only small fluctuations due to the

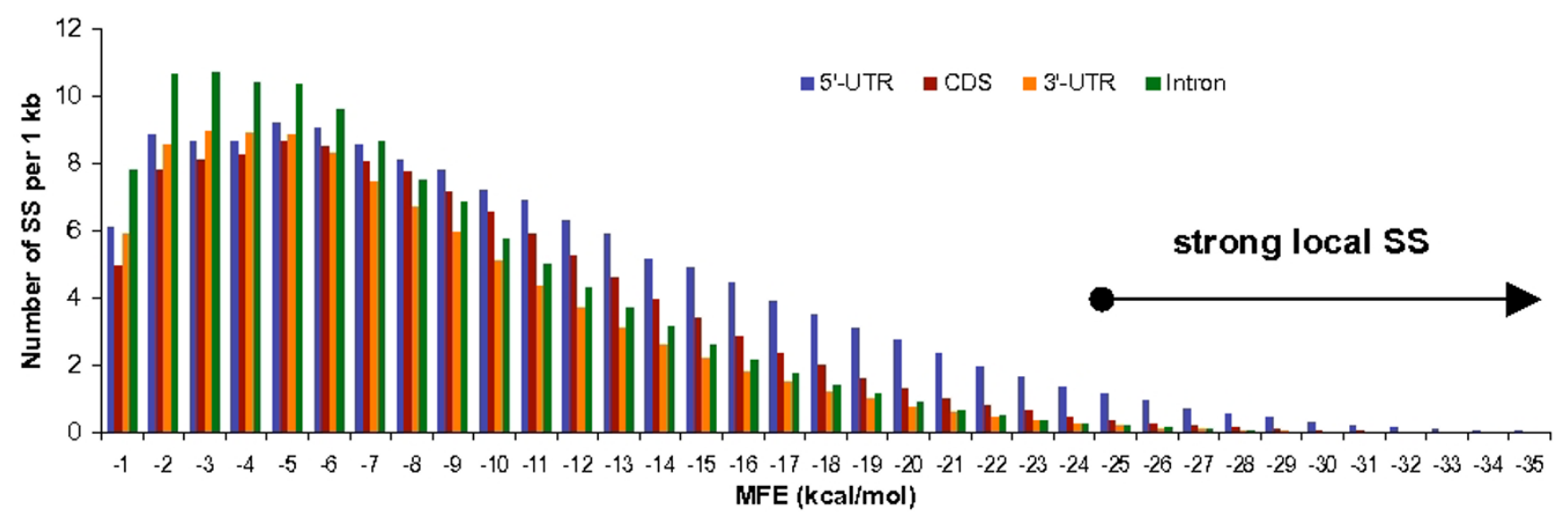

Figure I

Distribution of local SS with respect to folding energy in mRNA components and introns. Number of structures was measured within I $\mathrm{kcal} / \mathrm{mol}$ intervals and normalized by I,000 nucleotides of analyzed sequences. 
inherently random nature of the sequence generation process [see Additional file 1]. Pseudocode and further explanation of SRI-generator is provided for the reader as an additional file [see Additional file 2].

Figure 2A-C demonstrate the distribution of strong local SS in human 5'-UTR, 3'-UTR and intronic sequences and in their corresponding randomized counterpart sequences. Prediction of local SS was computed on a nonredundant sample of 11,315 human genes (see M\&M) by the RNALfold program [23]. All SRI-randomization was performed based on the tetramer frequency tables of the corresponding natural sequences. Tetramers reflect almost all short-range non-randomness since the major influence on SRI comes from adjacent bases [27]. Remarkably, the number of local SS in the natural sequences exceeds the number of structures of the same strength in the random sequences by a factor of at least 2 to 10 . Because we processed eleven thousand genes, the significance of this difference is unquestionable: the chi-square goodness-of-fit test gives a p-value less than 10-200. Having observed the difference between introns and their SRI-generated counterparts, we also examined sequences from intergenic regions (located between protein-coding genes and having an overall nucleotide composition similar to that of introns) and detected the same trend (Figure 2D). Moreover, the abundance of SS in natural sequences has no relation to genomic repetitive elements. Masking all human repeats with the RepeatMasker program [28] even mildly enhanced the difference in strong local SS between natural and SRI-generated sequences [see Additional file 3]. This observation simply reflects the fact that DNA repeats do not have an excess of strong SS, although some repeats have a distinct oligonucleotide composition and are enriched by $\mathrm{C} / \mathrm{G}$ bases (e.g. Alu family).

Protein-coding sequences (CDS) contain a profound $3 \mathrm{nt}$ periodicity and other non-randomness associated with translational properties [29]. All of this information would be lost in SRI-generated sequences. To overcome this problem we created CDS-generator, a public resource for the randomization of protein-coding sequences [26]. CDS-generator changes only the variable nucleotides in the third codon position, which do not change the coded amino acids. Additionally, CDS-generator maintains the codon and dicodon biases of a given set of natural coding

Table 2: Excerpt from oligonucleotide frequency table for 5'-UTRs of II,3 I 5 human genes and two SRI-generated counterparts. The entire dataset is presented in Additional file $\mathrm{I}$.

\begin{tabular}{|c|c|c|c|}
\hline Oligonucleotide & Human 5'-UTRs & Random I SRI-generated & Random 2 SRI-generated \\
\hline$A$ & 0.203 & 0.203 & 0.203 \\
\hline $\mathrm{T}$ & 0.201 & 0.201 & 0.2 \\
\hline C & 0.292 & 0.294 & 0.293 \\
\hline G & 0.303 & 0.303 & 0.303 \\
\hline$A A$ & 0.0506 & 0.0503 & 0.0506 \\
\hline AT & 0.0323 & 0.0323 & 0.0318 \\
\hline$A C$ & 0.0438 & 0.0437 & 0.0438 \\
\hline AG & 0.0771 & 0.0767 & 0.077 \\
\hline TA & 0.0258 & 0.0257 & 0.0256 \\
\hline TT & 0.0511 & 0.051 & 0.0508 \\
\hline $\mathrm{TC}$ & 0.0593 & 0.0591 & 0.0588 \\
\hline TG & 0.0653 & 0.0651 & 0.0651 \\
\hline CA & 0.0608 & 0.0609 & 0.0608 \\
\hline CT & 0.0726 & 0.0726 & 0.0725 \\
\hline $\mathrm{CC}$ & 0.0965 & 0.0973 & 0.0968 \\
\hline CG & 0.0627 & 0.0627 & 0.0629 \\
\hline GA & 0.0658 & 0.0661 & 0.0662 \\
\hline GT & 0.0453 & 0.045 & 0.0452 \\
\hline GC & 0.0932 & 0.0934 & 0.0936 \\
\hline GG & 0.0977 & 0.098 & 0.0984 \\
\hline$\ldots$ & $\ldots$ & $\ldots$ & $\ldots$ \\
\hline ACAA & 0.002558 & 0.002507 & 0.002544 \\
\hline ACAT & 0.002046 & 0.00205 & 0.002057 \\
\hline ACAC & 0.002775 & 0.002731 & 0.0028 \\
\hline ACAG & 0.004442 & 0.004362 & 0.00442 \\
\hline ACTA & 0.001396 & 0.001362 & 0.001388 \\
\hline ACTT & 0.002893 & 0.002899 & $0.00288 I$ \\
\hline АСТС & 0.003028 & 0.002958 & 0.003063 \\
\hline ACTG & 0.003961 & 0.003912 & 0.003954 \\
\hline$\ldots$ & $\ldots$ & $\ldots$ & $\ldots$ \\
\hline
\end{tabular}



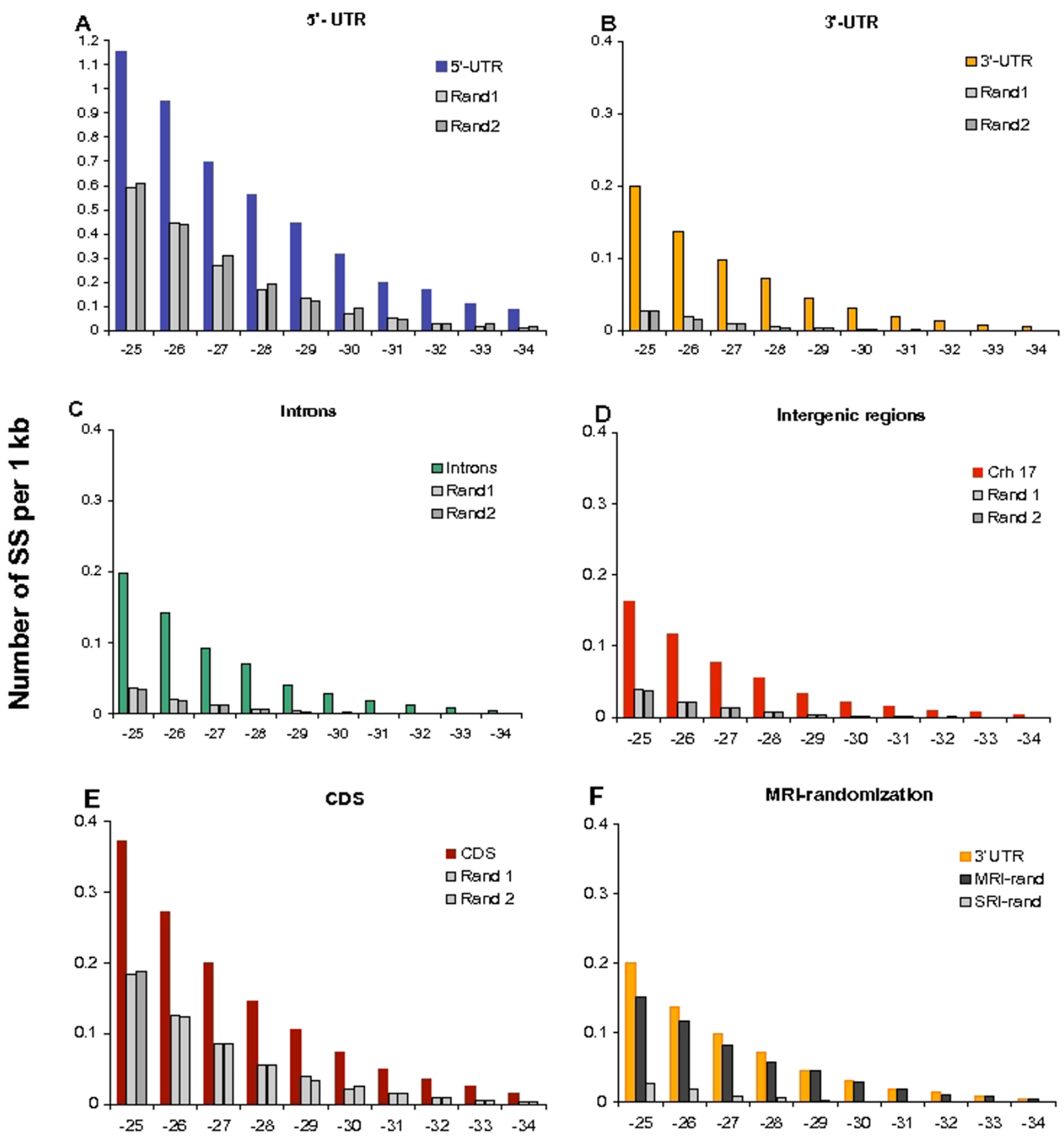

\section{MFE (kcal/mol)}

\section{Figure 2}

Distribution of strong local SS with respect to folding energy in mRNAs and genomic sequences. Number of structures was measured within I kcal/mol intervals and normalized by I,000 nucleotides of analyzed sequences. (A) 5'-UTRs (blue) and two independent SRI-generated sequences (gray); (B) 3'-UTRs (yellow) and two independent SRI-generated sequences (gray); (C) introns (green) and two independent SRI-generated sequences (gray); (D) intergenic regions from chromosome 17 (red) and two independent SRI-generated sequences (gray); (E) CDS (burgundy) and two independent CDS-generated sequences (gray); (F) 3'-UTRs (yellow), random MRI-generated counterpart sequences (black), and random SRI-generated counterpart sequences (gray). 
sequences. Thus, randomization by CDS-generator is much weaker than randomization by SRI-generator since it retains $>70 \%$ sequence identity between the natural and random sequences. On the other hand, maintaining a considerable level of sequence identity is useful because it preserves the major periodicity characteristics of the source coding sequences. Figure $2 \mathrm{E}$ demonstrates that natural coding sequences have twice the number of strong local SS as randomized sequences obtained by CDS-generator. The chi-square test confirms that the difference is statistically significant $\left(\mathrm{p}<10^{-200}\right)$.

\section{Mid-range inhomogeneity in natural genomic sequences}

To understand the observed abundance of strong local SS in mRNAs, we examined dozens of these structures in natural sequences, a typical example of which is shown in Figure 3. This structure, from a human 3'-UTR of the KIAA1751 gene, has an mfe of $-27.2 \mathrm{kcal} / \mathrm{mol}$ and represents a hairpin stem-loop configuration. The sequence of this SS is GC-rich (67\%) and is neighbored by several other alternating short AT-rich and GC-rich regions, as highlighted in Figure 3A. In contrast, such frequent alternation in GC-composition is practically absent in SRIgenerated sequences. Statistical examination revealed that strong local SS with mfe values in the range -25 to -30 $\mathrm{kcal} / \mathrm{mol}$ in human mRNAs have a mean GC-composition of $70 \%$, which is much higher than the average GC-composition of the mRNA, introns, or intergenic regions presented (Table 1). The observed GC-enrichment within strong local SS can be explained by thermodynamics (G$\mathrm{C}$ base pairs are about twice as strong as A-T pairs) and by combinatorics (random base-pairing is more frequent in GC-rich strands than when GC-composition is around $50 \%$ ). These notions have led to the hypothesis that natural sequences have a profound mid-range inhomogeneity, that is, they are enriched by short GC-rich regions (30$100 \mathrm{nt}$ ) alternating with adjacent AT-rich regions. In other words, we theorize the non-random clustering of $\mathrm{G} / \mathrm{C}$ and $\mathrm{A} / \mathrm{T}$ bases on the scale of $\sim 50$ nucleotides and the overabundance of such clusters in natural sequences.

To test this hypothesis we created a program named MRIanalyzer [26]. This program scans input sequences with a mid-range window size (the default, utilized for the results presented, is a 50 nt window). When the GC-content of the sequence in this window reaches the upper threshold, MRI-analyzer generates a blue top spike on the output graph (Figure 4). Similarly, when the GC-content of the window reaches the lower threshold, MRI-analyzer generates a red bottom spike. The upper and lower thresholds are flexible parameters defined by the user. MRI-analyzer output for natural and SRI-generated 3'-UTR and intronic sequences is shown in Figure 4. Here, the graph clearly shows a 35-fold enrichment of GC-rich ( $\geq 70 \%) 50$ nt-long regions in natural 3'-UTR sequences over the ran- domized SRI-generated sequences. For the $319 \mathrm{~kb}$ single extra-large intron in Figure 4C-D, GC-rich regions are enriched by a factor of 14 . Similar to the results shown in Figure 4, we observed comparable contrasts in GC-rich and GC-poor regions for 5'-UTR, intronic, and intergenic sequences for a wide range of scanning window sizes (301000 nt) (Figure 5). Additionally, MRI was generally observed with other base combinations and single bases (Figure 5). An example of the MRI pattern for GA- and CTrich regions is shown in Figures 6A and 6B; GT- and ACrich regions are presented in Figures $6 \mathrm{C}$ and 6D. The enrichment factor can be considered as the contrast between real and randomized sequences. We thus use the term "contrast" to refer to the ratio of the number of content-rich regions in a real sequence to the number in its SRI-generated counterpart. "Optimal contrast" is defined as the highest contrast observed over all thresholds for a given content type (for example, GC-content) and window size. Since we do not yet know the properties of midrange inhomogeneity in the human genome, we probed for the optimal contrast by repeating the above analysis for all thresholds starting from one standard deviation above (and below) the mean GC-content and proceeding until the number of GC-rich (or GC-poor) regions decreases to about ten. Figure 7 shows the distribution of optimal contrasts for all content types and a range of window sizes for the longest first intron of the DMD (dystrophin) gene.

\section{Association of MRI with the over-abundance of strong local SS}

Finally, we created a program named MRI-generator [26] for obtaining random sequences having the same oligonucleotide composition and also the same MRI pattern in GC-composition as a specified set of natural sequences. This program works by producing an excessively long SRIgenerated sequence and then discarding segments with intermediate GC-content to obtain the desired pattern of GC-rich and CG-poor regions. Thus, the output sequence from MRI-generator has a Genomic-MRI pattern of GCrich and GC-poor regions very similar to that of the natural sequence.

Comparison of natural sequences with their MRI-generated counterparts for each genomic sequence category (5'UTRs, 3'-UTRs, introns, intergenic regions, and CDS) shows that they each have approximately the same number (5-10\% difference) of strong local SS, as illustrated in Figure 2F for 3'-UTRs. This finding supports the conclusion that the abundance of strong SS in all parts of the mammalian genome (mRNA, introns, intergenic regions) is associated with the MRI of these sequences. 


\begin{abstract}
A
GCCUUCGAUGUUAAGUGACGGUUAAGCAAGAAAAUAUUCUUAUGUGCCACUAAGUGUUGUUUUUGGAGUGGACGAAAAUGUACAUUCAC GUUGACGCUAACCGAAGACUUGAAGGCACCUCCAGCAUUGGCCCAGCAGGCCCAGCACGGGCGGAGCCGCACAGGGCGGGGAAGAGGCU CUGCGGUGCCCCCUGGAGGGGCCGGGCUGCGGGGGGCUUCUGUCUAGCUCUGCAGCUGUGGCUGUUGUUAGACGCGCACCAGCGGGGAG AGGUGCCGAGAAACGGGCGGGCCCUGCCCUCCCCAUGCCUCCUGCCCUGCAGCCCGCCGCCAUCGGGAAUCGCUUGCGUCUCCUCCUUG CUUCUCUUGAUCGUUUUCCCACAUACGCAUCGAGAUCCAGAAACCUCCGUCCUCCUCCUGUCUGUCUUCGCGUGGUGCGCGUUCCGCAC GCCCUGCAGCUCAGCGCGGAGCUGGGCCGGUUCUCCACAGCCUGUGUGAGCCGCACUUCCGGUGUGAGCCGCACUUCCGGUGUGAGCCG CACUUCCGGUGUGAGCCGCACUUCCUGUCCUCACCACUGCGUGGCGUUUCCUUUCCUGAGAGAGCCUCGGGUUCUAGAUCCGUCCUUCU GCCAGUGCACACUUGGGAGCGUUCGUGGUACACACUGUUGCGAAGGCCGUUCUGCACUGCCCCUUGGCCUGUGUGUGAGUUCCCACAGG GCACCUGGCUGGGGCUCAUGCCUGCAUCACAGGGCAUAGCCAGCCUCACUGGCAUCAGCUAAUACCAAAGUGAGUUCUUUUUAUGUUGA GACAGGGUCUCUGUCGCCUAGGCUGGAGUGCAGUGGUGCAGUCACAGCUCACUGCAGCCUCGACCUCCCGGGCUCAAGCAAUCCUCCUG CCUCAGCUCCUGAGUAGCUGGAACUACAGGCACGCACCACCGCACCUGGCUAGUUUUUGUAUUUUUUUGUAGACACAGGGUUUCGCCAU GUUUCCCAGGCUGGUGUGGAACUCCUGAACUCAGGCUGUCUGCCCACCUCAGCCUCCCAAGGUGCUGGAUUACAGGCAUGAGCCACCUC GCCUAGCCCACCGAGUUCUUUUUCAAAGUGGUUGUGCAAGUUUACACUCCCACCAACAGUGUACAAGACUGUGCUGUCCAGCGUGGCUG UCACUGCCACAUCUGGCUAUUUAAACUUAAAUUGAUCCAAAUUAAAUAAUAGUGAAAAUUCAGGUCCUCCUCCACAAGGGCCACAUUUC ACAUGCUCCAUAGCGACCUGUGGGCAACGGCUGCCCUACUGGACAGCCCAGAAGCCCAGCUCUGCCUGGUCACAGAGGGUCCUACGGGG CCACACUGGGCUUGAGGGCCUUGCUUCACAUCCGCAGGAGCACGAGGUCACACGGCCCGGGGCUGCUUGUCUGGGGGGCCUGCACCACG GUCUGCUGUUGAGAUGUUGCUUUUUGGUUUCCCAGAUCACCAGUGAGAUUGCGUGUCUUUCCCUGGGCCCAUUUACAAGUUGUAUCUUC UUUGGUGUGACAUCUCUGUUUAAGGCUAUCCCUUGUUCUUCUGUGGGAUUGUCUGUCUUCUUCUCAUUCAUUCAUAGGAGCUCUUCAUG CACCCAGAUUCCAGCCCUCCUCCCUGAGGAAUGUUGCAGGUGCUGCCUCCGAGUCUGUGGCUGGGCCUUCCUCUCCCUUUAUGACGUCU UUUCUCAAAUCAAAGUUCUUCAGUCCAGUCUUUGCAUAUUUACCAGUCAUUUCUAUUGGAAUUUGUUUUUUAUGAUUUUUAGGCAAUUU UUUUCUCCUCCAGGGUCAUAAAGACAUAGGACAUCGCUUUUGUGUUGAGGUGUAUGAUCCACCUCAAAUUCACUCCUGCUUGUGGUGGA AGUCAGGGCUGAGGCUCAUUUCCCCUGGUGCGUGUUUGUGUAUUCCACGGCACAAGUGGACUCUGCUAUCCCAUUCGAUCCAUUCGUUG UCCGUGUCAAAAAUCAAUUGACCAUCCAGGCACAGUGGCUCAUGCCUGUAAUCCCAGAACUUUGGGAGGCCGAGGCAGGAGGAUCACUU GAGGUCAAGAGUUUGAGACCAGCCUGGCCAACAUGGUGAAACCCCAUUUCUACUAAAAAUACAAAAAAUUAGCCAGGCGUGGUGGCGGG CGCCUGUAAUCCCAGCUACUUGGGUGGCUGAGGCAAGAGAAUUGCUUGAACCCGGGAGGCGGAGGUUGCAGUGAGCUGAGAUCGCGCCA UUGCACUCCAGCCUGGCCUAUAAGAGUAAAAUACUGUCUC
\end{abstract}

$.((((((((((((\ldots \ldots))))))(((((\ldots))))) \ldots))))))) \quad(-27.20) 1298$

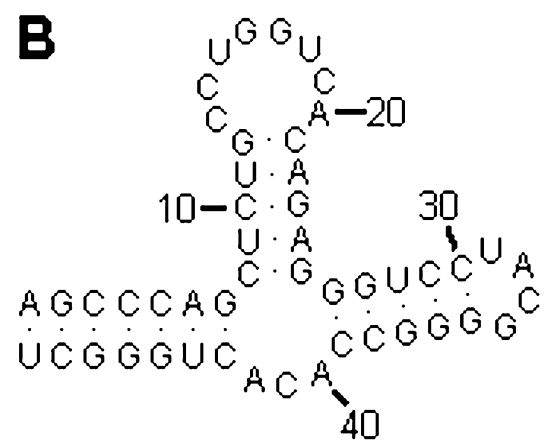

\title{
Figure 3
}

Example of a strong local SS in the 3'-UTR of the human KIAAI 75 I gene [GenBank:NM_00 I080484]. (A) Nucleotide sequence of the entire $3^{\prime}-$ UTR region in which a segment exemplifying a strong local SS $(\mathrm{mfe}=-27.2 \mathrm{kcal} / \mathrm{mol})$ is shown in red and its schematic base-pairing is shown in dot-bracket notation [23] below the sequence. Other GC-rich regions are highlighted in blue and GC-poor regions are underlined. (B) 2-D representation of this strong SS.

\section{DNA repetitive elements and genomic MRI}

Even though human interspersed repeats do not show an excess of strong SS as discussed above, they do influence the patterns of MRI [see Additional file 4]. The figure in additional file 4 illustrates the MRI patterns of the extralarge first intron of the DMD gene (see Figure 4) after masking its repetitive elements by RepeatMasker. Unsurprisingly, the number of MRI regions in the masked sequence is a fraction of those in its non-masked counterpart. The masked sequence contains $41 \% \mathrm{~N}$ 's instead of $\mathrm{A}$, $\mathrm{G}, \mathrm{C}$, or T bases. The current version of MRI-analyzer skips a window containing any non-A, G, C, or T character. For a proper comparison of MRI patterns in a masked sequence, one should compare it to the SRI-generated random sequence based on the masked sequence. Such random sequences contain the same number of N's at exactly 

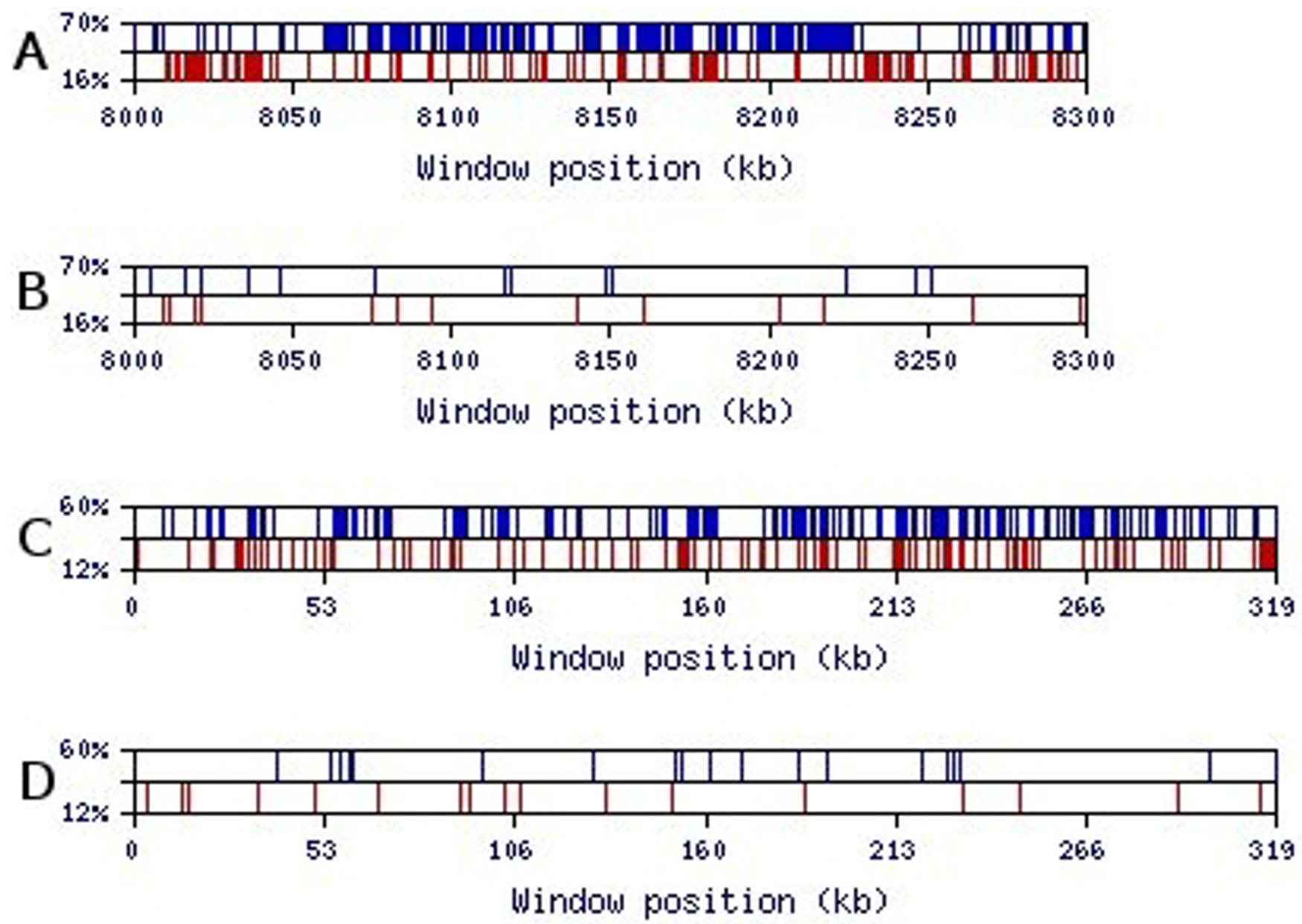

Figure 4

Visualization of MRI-analyzer output for GC-composition of two 300 kb samples using a 50-nt window. Upper and lower thresholds are specified on the $y$-axis as a percentage of the window size. (A) A sequential sample of human 3'-UTRs from chromosomes I and 2 (EID ids I745_NT_004487 through 2327_NT_022184); (B) a random SRI-generated set based on the tetramer oligonucleotide frequency table of II,3I5 human 3'-UTR sequences. (C) The 319 kb sequence of the first extralarge intron of the DMD gene; (D) a random SRI-generated set based on the tetramer oligonucleotide frequency table of the first intron of the DMD gene.

at the same positions as the natural masked sequence. The figure in Additional file 4 demonstrates that the masked sequence of the first $D M D$ intron has 3 to 12 times the number of MRI peaks compared to its random counterpart. This particular example with the DMD intron presents an AT-rich sequence ( $67 \%$ of $A+T)$, which is typical for extra-large introns [17]. Accordingly, we set the upper threshold for GC-composition to $60 \%$ in studying this sequence. Under such conditions, we observe GC-rich MRI peaks overlapping various portions of Alu-repeats. This overlapping of MRI regions with repetitive elements seems to depend on the threshold used and the G+C-composition of the region under analysis.

\section{Discussion}

We have demonstrated an association between MRI in GC composition and the abundance of strong SS in genomic sequences. There are at least two possible interpretations of these results. First, one can argue that MRI causes the abundance of strong SS. The second possibility is that selection for strong SS was the reason for the appearance of MRI. Both views have merit and we thus include a discussion of the supporting evidence.

Central to this discussion is the observation that MRI exists not only in mRNA sequences but also in introns and intergenic regions. If selection were limited to transcripts or to mature mRNAs, there would be no way for evolution to directly drive the creation of SS in non-transcribed 

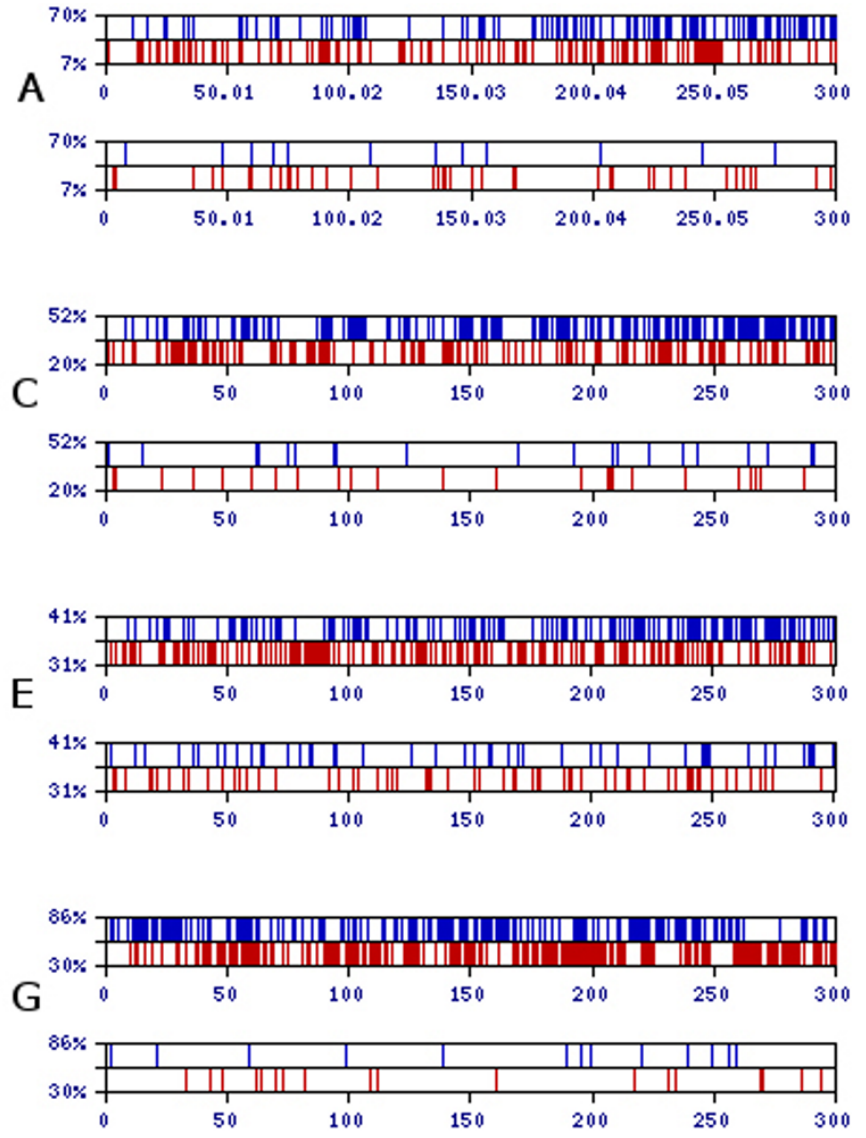

Window position (kb)
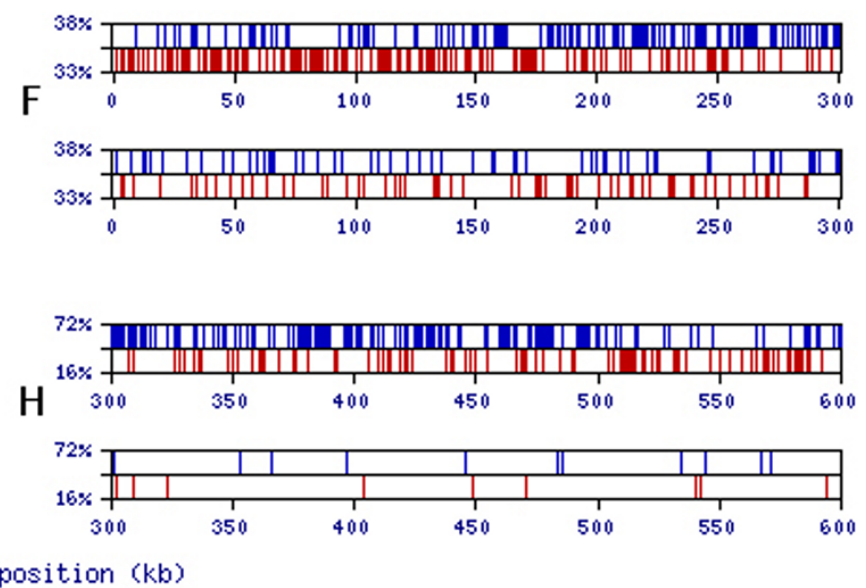

Figure 5

Comparison of MRI-analyses of GC-content for various window sizes and genomic contexts. (A-F) The $319 \mathrm{~kb}$ sequence of the first intron from the DMD gene, and its SRI-generated counterpart, analyzed for optimal visual contrast over a range of window sizes $(30,50,100,200,500,1000)$ (cf. Figures 4 and 7); (G) The first $300 \mathrm{~kb}$ of a sample of human 5'-UTRs and its SRI-generated counterpart using a window size of $50 \mathrm{nt}$; $(\mathbf{H})$ The $300 \mathrm{~kb}$ subset from a sample of intergenic sequences from human chromosome 17 and a corresponding SRI-generated sequence using a window size of $50 \mathrm{nt}$.

regions. This would leave MRI in GC composition as a potential mediator of strong SS enrichment. However, some experimental evidence suggests that much more of the genome is transcribed than previously thought [30]. It has also been suggested for some time that SS play a role in the initiation of recombination. This theory predicts positive selection for SS throughout genomes and especially within introns and intergenic regions [31-33]. Moreover, studies of coding sequences in mammals have found that synonymous substitutions tend to increase the strength of SS and regulate mRNA stability [34-36]. Thus, SS could have emerged first due to selection for DNA hairpins to facilitate homologous recombination and for stable mRNA SS signals, yielding MRI in GC content as a byproduct. On the other hand, MRI is also observed for AGand GT-content as well as for the individual nucleotides (see Figures 6 and 7), so it is also possible that selection for MRI is a fundamental force driving genome organization and composition.

It is of special interest to investigate possible biological roles for MRI in the structural and functional organization of mammalian genomes. To address this important issue, we have studied 3.3 million point mutations occurring over the last 10 million years in humans and over 3.9 million SNPs in the MRI-regions and outside them. These results will be detailed in our next publication (under preparation). Based on the preliminary results of these investigations, we can state that MRI patterns are formed by a combination of processes. Some patterns (e.g. A+Trich regions) are like cellular automata, based on nonselection biases in nucleotide changes at genomic regions with specific base compositions, while other patterns are formed by a strong fixation bias (presumably positive 

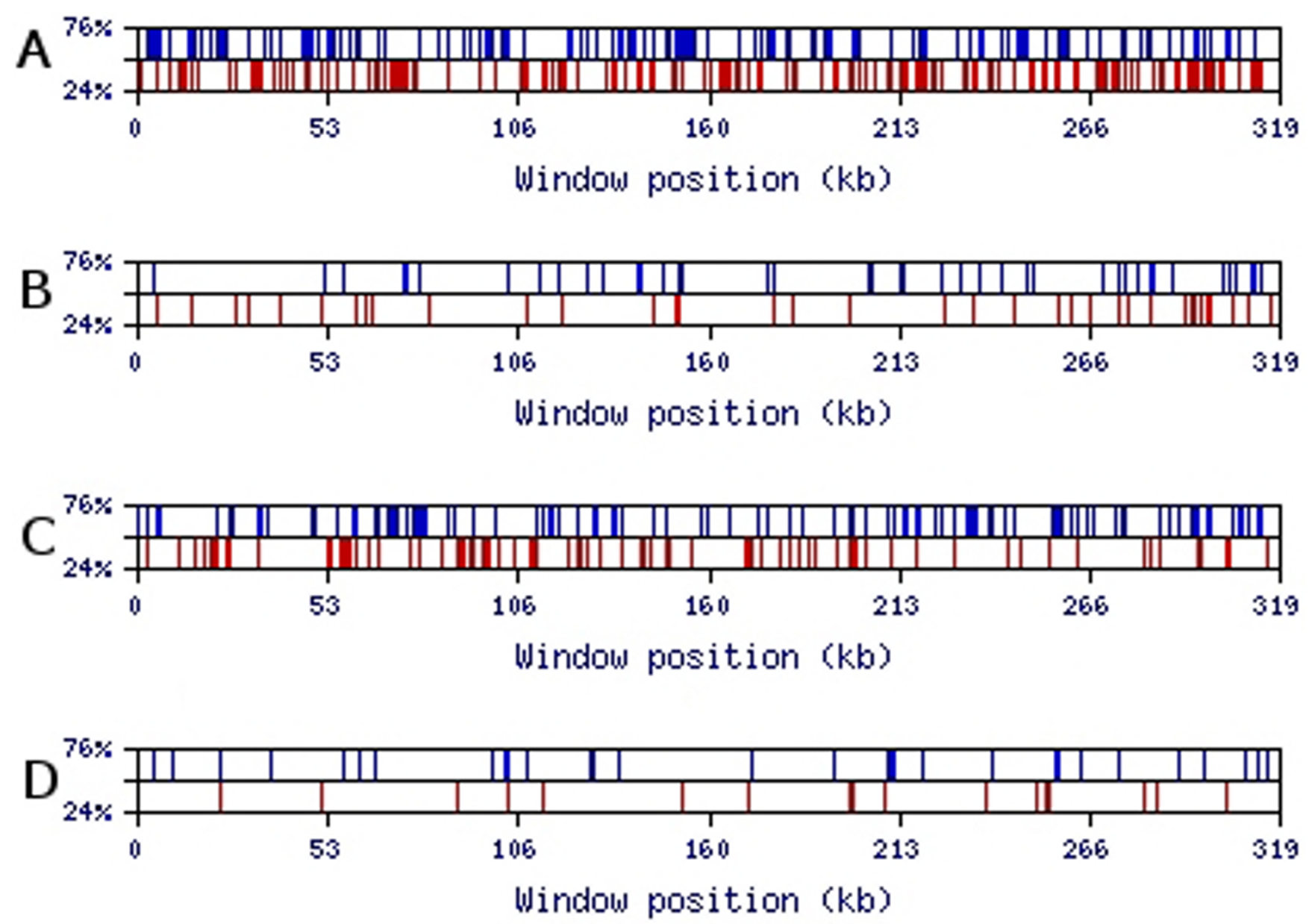

Figure 6

Visualization of MRI-analyzeroutput for AG- and GT-compositions of 319 kb sequence of the first intron of the DMD gene using a $\mathbf{5 0}$ nt window. Upper and lower thresholds are specified on the $y$-axis as a percentage of the window size. (A) AG-rich and AG-poor regions of the DMD intron; (B) AG-rich and AG-poor regions of the corresponding random SRI-generated set based on the tetramer oligonucleotide frequency table of the DMD intron; (C) GT-rich and GT-poor regions of the DMD intron; (D) GT-rich and GT-poor regions of the corresponding random SRI-generated set based on the tetramer oligonucleotide frequency table of the DMD intron.

selection of functional regions) that preserve particular base enrichments in corresponding regions (e.g. G+C-, purine-, and pyrimidine-rich). These forces drive midrange non-randomness, shaping the human genome and potentially imparting additional layers of organizational complexity.

Indeed, an important feature of the human genome is that its vast array of genes is differentially expressed in hundreds of different cell types and subtypes. Moreover, at different stages of development and in response to diverse extracellular stimuli, gene expression must be finely tuned. To perform the enormous task of creating a human body composed of trillions of cells, the genome must contain a vast number of signals for gene regulation, the majority of which have yet to be discovered. We hypothesize that MRI could represent a novel class of genomic signals, based on overall composition and clustering of nucleotides rather than particular sequence motifs. To facilitate the testing of this hypothesis, we created a free, public Internet resource called "Genomic MRI" that allows one to run all programs described here without any programming knowledge. Additionally, all of these programs are freely available for downloading and off-line usage, primarily for computational biologists.

\section{Methods}

The programs SRI-analyzer, SRI-generator, MRI-analyzer, MRI-generator, and CDS-generator are available via our 


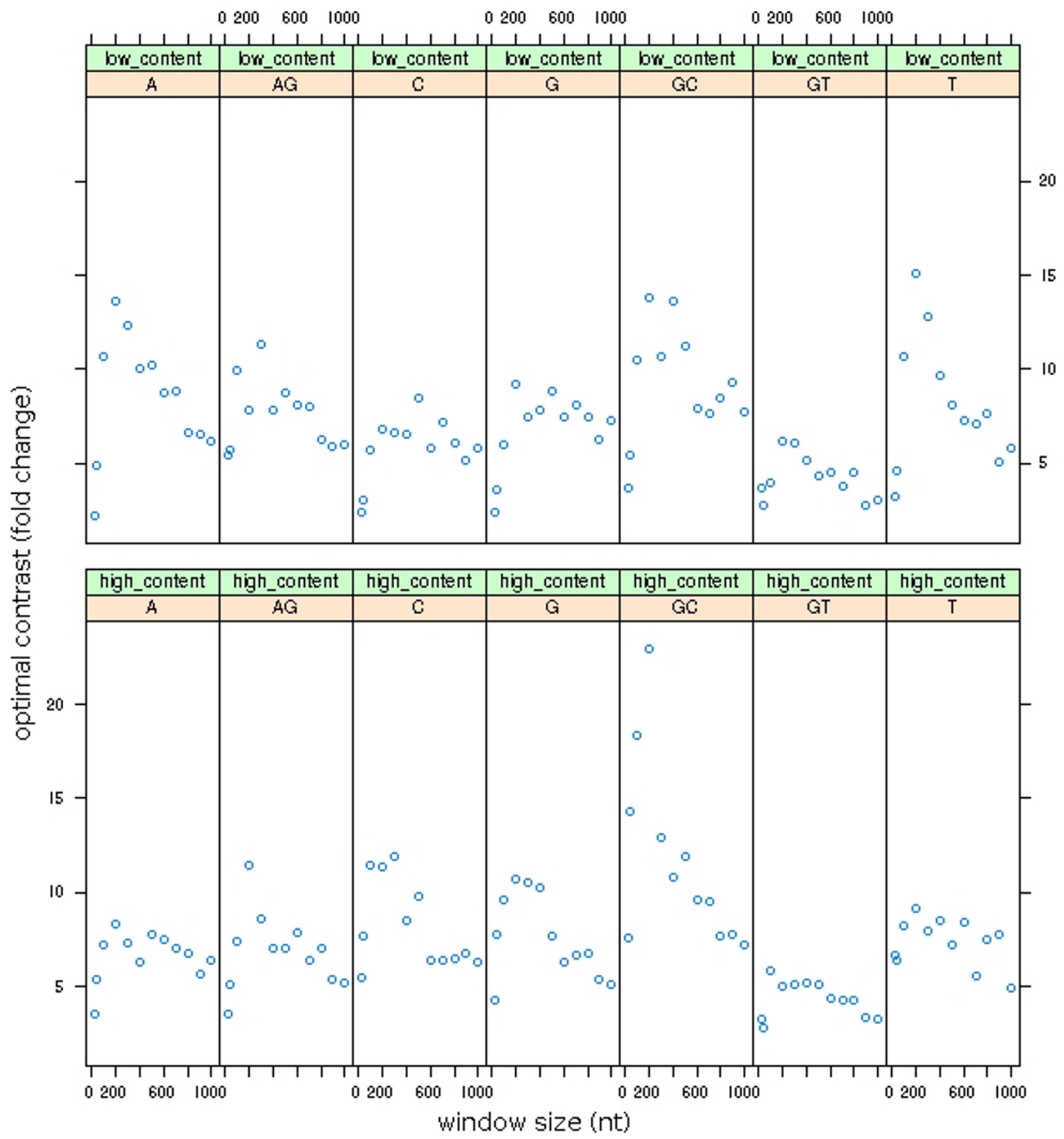

Figure 7

Optimal contrasts for all content types over a range of window sizes. This figure is the "XY conditioning plot" (from the program Rcmdr I.2) of the optimal contrasts (see text) for regions of high and low content for all seven possible content types over a range of window sizes $(30,50,100,200,300, \ldots 1000)$. The sample sequence is the $319 \mathrm{~kb}$ first intron from the DMD gene. The SRI-generated counterpart is constructed from the tetramer frequency table derived from the intron. 
website. A link to the current location of the website will be maintained at our departmental project site [26].

\section{Sequence randomization algorithm (SRI-generator)}

There are several possible approaches for randomizing nucleotide sequences while maintaining their N-mer oligonucleotide frequency composition. The simplest approach would be to randomly choose N-mer oligonucleotides based on their frequency composition and tile them one after the other. However, this approach does not necessarily preserve the frequencies of shorter length oligonucleotides that one may observe in natural sequences. For example, the random concatenation of $\mathrm{N}$ mers as tiles artificially introduces dinucleotide composition bias created from the border of two adjacent oligonucleotide tiles - producing an overrepresentation of $\mathrm{CpG}$ dinucleotides and the like that do not match the SRI natural sequences. Therefore we chose a different approach which generates a randomized sequence one nucleotide at a time moving in a $5^{\prime}$ to $3^{\prime}$ direction.

We generate randomized sequences in the following manner. First we choose the largest oligonucleotide size (N) that is sufficiently sampled. In practice, this means avoiding sizes for which some of the oligonucleotides are never encountered in the input sequence (i.e. occur with zero frequency). Throughout our study we used 4-mer oligonucleotides $(\mathrm{N}=4)$ because they were consistently well sampled across all of our input sequences, including a single large intron in the Figure 2C. The starting oligonucleotide is chosen at random, abiding by the frequency table for oligonucleotides of the chosen size $(\mathrm{N})$. Next, we observe the last ( $\mathrm{N}-1$ ) bases of our sequence, and append a base to the 3' end, following the N-mer oligonucleotide frequencies. For example, if $\mathrm{N}=4$ and GTC were the last three bases in the growing random sequence, the frequencies of GTCA, GTCT, GTCC, and GTCG would be used in randomly adding the next base. For instance, suppose these four oligomers have relative frequencies of $0.5,0.1,0.2$, and 0.2 , respectively. Then the random number generator will append 'A' with a probability of 0.5 , ' $\mathrm{T}$ ' with a probability of 0.1 , 'C' with a probability of 0.2 , and ' $G$ ' with probability of 0.2 . This final step is then repeated until the randomized sequence reaches the length of the input sequence. In contrast to the tiling method, our approach preserves the frequencies of short oligonucleotides in addition to preserving the N-mer frequency composition.

Finally, we made our SRI-generator work properly with sequences that have masked repetitive elements (where all sequences of DNA repeats are replaced by N's by the RepeatMasker program). Any non-A, T, C, or G bases are copied from the source sequence over the output sequence. The random sequences thus contain the same number of N's (or other non-A, T, C, or G bases) in the same positions as in the natural sequences provided as input.

The pseudocode for SRI-generator is presented in Additional file 2, while the source code (written in Perl) is freely available from our project website [26].

\section{CDS-generator}

Several sophisticated algorithms are already available for the randomization of coding sequences $[37,38]$. However, here we used our own randomization approach developed by AF in 2001 while working on a contextdependent codon bias project in the Walter Gilbert lab [29]. We stayed with our program because we are familiar with the peculiarities of this type of randomization. In addition our approach gets the dicodon distribution of randomized sequences very close to that of the natural CDS.

\section{Program notes}

1) We observe a gradual diminution of the difference between real and randomized sequences when using progressively larger oligonucleotides with the randomized sequence generation programs (SRI-generator and MRIgenerator). The difference is not considerable, but it is noticeable. Therefore, we recommend the use of longer oligonucleotides in the construction of randomized sequences - to maximize the retention of short-range inhomogeneity - as long as the rarest oligonucleotide in the corresponding frequency table occurs at least ten times. We use tetramer frequency tables throughout the manuscript for the sake of consistency and since they can safely be used for analyses of individual loci having as little as $100 \mathrm{~kb}$.

WARNING: In MRI-generator it is easy to shift the nucleotide content level of generated sequences by using thresholds that do not balance the number of content-rich and content-poor regions. One must experiment with the thresholds and use SRI-analyzer to confirm that the content of the MRI-generated sequence approximates that of the source sequence.

2) The graphical output provided with the online version of MRI-analyzer serves only as a quick visual aid. The true output is represented by large tab-delimited files containing a record for each window in the analysis. Each record contains flags indicating a content-rich or content-poor window and, for those records where one of the thresholds has been crossed, the corresponding sequence.

3) All programs are written in Perl and may be freely downloaded from the website. They are licensed under version 3 of the GNU General Public License (GPL). 
4) The RNALfold program from version 1.6.1 of the Vienna RNA package was utilized locally on our computers with default parameters.

\section{Source for gene sample set}

Our sample of 11,315 non-redundant human genes (with $<50 \%$ sequence identities between each other) was obtained from the human Exon-Intron Database, release 35p1 [39]. Samples of intergenic regions were obtained from Genbank human genome files build 36 based on the records from the Feature Tables. We used only plus strands for calculations because there are only fluctuation differences between plus and minus strands in the noncoding regions of mammalian genomes. Also, plus and minus strands have the same $\mathrm{G}+\mathrm{C}$ and $\mathrm{A}+\mathrm{T}$ compositions. All these samples are available from our departmental project site [26].

\section{Abbreviations}

SS: secondary structure(s); MRI: mid-range inhomogeneity; SRI: short-range inhomogeneity; UTR: untranslated region; CDS: coding sequence(s); nt: nucleotide(s); kb: kilobase(s).

\section{Authors' contributions}

JMB, TW, TD, JS, SA, SKR, SS were responsible for computational processing of all datasets and creation of all described programs. JMB and SS also created the Genomic MRI web resource. AF supervised the project, provided guidance and wrote the draft. All authors have read and approved the final manuscript.

\section{Additional material}

\section{Additional file 1}

Complete oligonucleotide frequency table for natural 5'-UTRs of 11,315 human genes and two SRI-generated counterpart sequences. This file is also available from our departmental project web page [26].

Click here for file

[http://www.biomedcentral.com/content/supplementary/1471-

2164-9-284-S1.xls]

\section{Additional file 2}

Pseudocode and further explanation of the algorithm used in SRI-generator. This file is also available from our departmental project web page [26].

Click here for file

[http://www.biomedcentral.com/content/supplementary/14712164-9-284-S2.pdf]

\section{Additional file 3}

Distribution of strong local SS with respect to folding energy in masked intergenic regions of human chromosome 17. Number of structures was measured within $1 \mathrm{kcal} / \mathrm{mol}$ intervals and normalized by 1,000 nucleotides of analyzed sequences. Results are shown for intergenic regions from chromosome 17 (red) and two independent SRI-generated sequences (gray). All sequences are masked with the RepeatMasker program. (A) Results with masked positions ('N's) retained, which reduces the density of secondary structures; (B) results with masked positions ('N's) removed in order to compare with the graphs in Figure 2.

Click here for file

[http://www.biomedcentral.com/content/supplementary/1471-

2164-9-284-S3.pdf]

\section{Additional file 4}

Comparison of MRI-analyses of GC-, AG- and GT-content with a 50 nt window in masked DMD intron 1. The first intron of the DMD gene was masked using the RepeatMasker program. SRI-generated counterpart sequences retain all masked positions. In each figure the MRI pattern for the natural sequence and the randomized counterpart is shown above and below, respectively: (A) analyzed for MRI in GC-composition; (B) analysis for MRI in AG-composition; (C) analysis for MRI in GT-composition. Click here for file

[http://www.biomedcentral.com/content/supplementary/14712164-9-284-S4.jpeg]

\section{Acknowledgements}

This project is supported by NSF Career award MCB-0643542. We thank Peter Bazeley, University of Toledo, for his computational support and discussion of our algorithms.

\section{References}

I. Buratti E, Baralle FE: Influence of RNA secondary structure on the pre-mRNA splicing process. Mol Cell Biol 2004, 24: $10505-105 \mid 4$

2. Antequera F: Structure, function and evolution of $\mathrm{CpG}$ island promoters. Cellular and Molecular Life Sciences 2003, 60:1647-I658.

3. Marashi SA, Eslahchi C, Pezeshk H, Sadeghi M: Impact of RNA structure on the prediction of donor and acceptor splice sites. BMC Bioinformatics 2006, 7:297.

4. Kozak M: Regulation of translation via mRNA structure in prokaryotes and eukaryotes. Gene 2005, 36 I:13-37.

5. Pickering $B M$, Willis $A E$ : The implications of structured 5' untranslated regions on translation and disease. Semin Cell Dev Biol 2005, 16:39-47.

6. Chabanon H, Mickleburgh I, Hesketh J: Zipcodes and postage stamps: mRNA localisation signals and their trans-acting binding proteins. Brief Funct Genomic Proteomic 2004, 3:240-256.

7. Chen JM, Ferec C, Cooper DN: A systematic analysis of diseaseassociated variants in the $3^{\prime}$ regulatory regions of human protein-coding genes II: the importance of mRNA secondary structure in assessing the functionality of 3' UTR variants. Hum Genet 2006, I 20:30I-33.

8. Svoboda P, Di Cara A: Hairpin RNA: a secondary structure of primary importance. Cell Mol Life Sci 2006, 63:90I-8.

9. Meyer IM, Miklos I: Statistical evidence for conserved, local secondary structure in the coding regions of eukaryotic mRNAs and pre-mRNAs. Nucleic Acids Res 2005, 33:6338-6348.

10. Shabalina SA, Ogurtsov AY, Spiridonov NA: A periodic pattern of mRNA secondary structure created by the genetic code. Nucleic Acids Res 2006, 34:2428-2437.

II. Seffens W, Digby D: mRNAs have greater negative folding free energies than shuffled or codon choice randomized sequences. Nucleic Acids Res 1999, 27:1578-1584. 
12. Workman C, Krogh A: No evidence that mRNAs have lower folding free energies than random sequences with the same dinucleotide distribution. Nucleic Acids Res 1999, 27:4816-4822.

13. Karlin S, Campbell AM, Mrázek J: Comparative DNA analysis across diverse genomes. Annu Rev Genet 1998, 2:185-225.

14. Karlin S, Burge C: Dinucleotide relative abundance extremes: a genomic signature. Trends Genet 1995, I I:283-290.

15. Campbell A, Mrázek J, Karlin S: Genome signature comparisons among prokaryote, plasmid, and mitochondrial DNA. PNAS 1999, 96:9184-9189.

16. Bernardi G: The Vertebrate Genome: Isochores and Evolution. Mol Biol Evol 1993, 10:186-204.

17. Bernardi G: The neoselectionist theory of genome evolution. PNAS 2007, 104:8385-8390

18. Duret L, Eyre-Walker A, Galtier N: A new perspective on isochore evolution. Gene 2006, 385:71-74.

19. Gardiner-Garden M, Frommer M: CpG islands in vertebrate genomes. J Mol Biol 1987, 196:26I-282.

20. Takai D, Jones PA: The CpG island searcher: a new WWW resource. In Silico Biol 2003, 3(3):235-240.

21. Hackenberg M, Previti C, Luque-Escamilla PL, Carpena P, MartínezAroza J, Oliver JL: CpGcluster: a distance-based algorithm for CpG-island detection. BMC Bioinformatics 2006, 7:446.

22. Mathews DH: Predicting a set of minimal free energy RNA secondary structures common to two sequences. Bioinformatics 2005, 21:2246-2253.

23. Hofacker IL: Vienna RNA secondary structure server. Nuc Acids Res 2003, 31:3429-3431.

24. Kishore S, Stamm S: The snoRNA HBII-52 regulates alternative splicing of the serotonin receptor 2C. Science 2006, 3 I I:230-232.

25. Griffiths-Jones S, Grocock RJ, van Dongen S, Bateman A, Enright AJ: miRBase: microRNA sequences, targets and gene nomenclature. Nucl Acids Res 2006, 34:DI40-DI44.

26. All described programs are freely available via our web site: Genomic Mid-Range Inhomogeneity. [http://hsc.utoledo.edu/ depts/bioinfo/gmri/].

27. Karlin S, Mrázek J: What drives codon choices in human genes? J Mol Biol 1996, 262:459-472.

28. Smit AFA, Hubley R, Green P: RepeatMasker Open-3.0. 2004 [http://www.repeatmasker.org].

29. Fedorov A, Saxonov S, Gilbert W: Regularities of contextdependent codon bias in eukaryotic genes. Nucleic Acids Res 2002, 30:1 192-1197.

30. Kapranov P, Willingham AT, Gingeras TR: Genome-wide transcription and the implications for genomic organization. Nat Rev Genet 2007, 8:413-423.

31. Forsdyke DR: A stem-loop kissing model for the initiation of recombination and the origin of introns. Mol Biol Evol 1995, 1 2:949-958.

32. Forsdyke DR: Stem-loop potential in MHC genes: $\mathbf{A}$ new way of evaluating positive Darwinian selection? Immunogenetics 1996, 43: 182-189.

33. Forsdyke DR: An alternative way of thinking about stem-loops in DNA. A case study of the human GOS2 gene. J Theor Biol 1998, 192:489-504.

34. Chamary JV, Hurst LD: Evidence for selection on synonymous mutations affecting stability of mRNA secondary structure in mammals. Genome Biol 2005, 6:R75.

35. Duan JB, Wainright MS, Comeron JM, Saitou N, Sanders AR, Gelernter J, Gejman PV: Synonymous mutations in the human dopamine receptor D2 (DRD2) affect mRNA stability and synthesis of the receptor. Hum Mol Genet 2003, I 2:205-2 I6.

36. Nackley AG, Shabalina SA, Tchivileva IE, Satterfield K, Korchynskyi O, Makarov SS, Maixner W, Diatchenko L: Human catechol-O-methyltransferase haplotypes modulate protein expression by altering mRNA secondary structure. Science 1996, 314:1930-1933.

37. Katz L, Burge CB: Widespread selection for local RNA secondary structure in coding regions of bacterial genes. Genome Res 2003, 9:2042-205।.

38. Down T, Leong B, Hubbard T]: A machine learning strategy to identify candidate binding sites in human protein-coding sequence. BMC Bioinformatics 2006, 7:419. Fedorov A, Saxonov S, Gilbert W: Regularities of context-dependent codon bias in eukaryotic genes. Nucleic Acids Research 2002, 30: I I $92-1$ I 97
39. Shepelev V, Fedorov A: Advances in the Exon-Intron Database (EID). Briefings in Bioinformatics 2006, 7:178-85.
Publish with Biomed Central and every scientist can read your work free of charge

"BioMed Central will be the most significant development for disseminating the results of biomedical research in our lifetime. "

Sir Paul Nurse, Cancer Research UK

Your research papers will be:

- available free of charge to the entire biomedical community

- peer reviewed and published immediately upon acceptance

- cited in PubMed and archived on PubMed Central

- yours - you keep the copyright 